XIV Simpósio Nacional de Sistemas Prediais

Gestão, Eficiência e Sustentabilidade

\title{
TÉCNICAS DE AUDITORIA DO CONSUMO DE ÁGUA EM EDIFICAÇÕES
}

\section{Building water audit techniques}

\author{
SANTANA, Livia Ferreira'; SANT'ANA, Daniel Richard²
}

Recebido em 15 de julho de 2021, aprovado em 06 de setembro de 2021, publicado em 18 de novembro de 2021

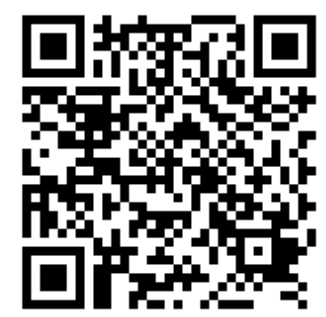

Palavras-chave:

Auditoria de água,

Consumo de água,

Usos finais de água.

Keywords:

Water audit,

Water consumption,

Water end-uses.

\begin{abstract}
RESUMO: Diferentes técnicas de auditoria de água podem ser usadas para caracterizar os usos finais de água em edificações. A escolha das técnicas mais apropriadas é essencial para planejar uma auditoria eficaz em diferentes tipos de edifícios. Com isso em mente, este trabalho propôs classificar e analisar as diferentes técnicas de auditoria da água com base em uma revisão dos métodos delineados por estudos anteriores. Os resultados indicam que a maioria dos estudos usa mais de uma técnica de auditoria de água para coletar informações sobre o uso e consumo de água em edifícios. Análise documental e medições gerais são técnicas simples que ajudam a compreender o perfil do consumo de água das edificações e gerar indicadores de consumo predial. Entrevistas e questionários são técnicas de baixo custo que fornecem dados que podem ser usados para estimar os usos finais de água. Medições setorizadas fornecem informações sobre o consumo de água para determinadas atividades, ambientes ou trechos da rede hidráulica, enquanto medições específicas são capazes de fornecer dados por ponto de uso. Embora sejam capazes de fornecer dados precisos, apenas $8 \%$ dos estudos analisados fazem uso da técnica de análise de traço e $19 \%$ utilizam sensores de fluxo para caracterizar os usos finais de água em edificações, provavelmente devido à sua complexidade e custo elevado.
\end{abstract}

ABSTRACT: Different water auditing techniques can be used to characterize building water end-uses and consumption. Choosing the most appropriate techniques is essential to plan an effective water audit for different types of buildings. With this in mind, this paper set out to classify and analyze different water auditing techniques based on a review of the methods designed by previous studies. Results indicate that most studies use more than one water auditing technique to collect information regarding building water use and consumption. Documental analysis and general measurements from meter readings are simple techniques that helps to understand the profile of building water consumption and to generate water consumption indicators. Interviews and questionnaires are low-cost techniques that provide qualitative data that can be used to estimate water use. Sectorized measurements provide information regarding water consumption for certain activities, spaces or sections of plumbing network, whilst specific measurements are capable of providing water consumption data per fixture use. Measurements make use of water meters, flow rate trace equipment or water flow sensors. These might be connected to data logging and telemetry equipment to register water use events. Even though they are capable of providing precise data, only $8 \%$ of the analyzed studies makes use of the flow rate trace technique or water flow sensors (19\%) to characterize building water end-use, probably due to its complexity and high costs.

\section{CONTATO DOS AUTORES:}

${ }^{1}$ SANTANA, Livia Ferreira: Grupo de Pesquisa Água \& Ambiente Construído, Instituto Federal de Goiás, livia.santana@ifg.edu.br

2 SANT'ANA, Daniel Richard: Grupo de Pesquisa Água \& Ambiente Construído, Universidade de Brasília, dsantana@unb.br 


\section{INTRODUÇÃO}

Alcantara et al. (2021, p.143) definem auditoria de água como um "processo documentado na avaliação quantitativa e qualitativa dos fluxos de água dentro de um determinado domínio". Em sua essência quantitativa, o volume de água utilizada em diferentes tipos de uso é examinado (auditoria do consumo de água). Já sua essência qualitativa avalia as características físicas, químicas e bacteriológicas dos volumes de entrada e saída dentro de um determinado domínio (auditoria da qualidade de água). Auditoria de água pode ser realizada em diferentes escalas e cenários, em sistemas naturais (como bacias hidrográficas, rios e lagos) ou no ambiente construído (em sistemas hidráulicos urbanos e prediais).

Em edificações, a auditoria do consumo de água é capaz de realizar um diagnóstico do desempenho do sistema predial, gerando indicadores de consumo, vazão de equipamentos hidrossanitários, frequência e tempo de uso, além de extrair informações sobre hábitos de uso e rotinas de manutenção predial, higienização de ambientes e irrigação paisagística (ALCANTARA et al., 2021). Essas informações são essenciais para o dimensionamento de sistemas prediais de água (HONORATO e CAMPOS, 2019), desenvolvimento de equipamentos economizadores de água (ROCHA et al., 1998), análise e avaliação de programas de conservação de água (SANT'ANA e MAZZEGA, 2018), previsão de demanda de água (DEOREO et al., 1996), gestão de recursos hídricos e criação de políticas públicas (MAZZEGA et al., 2019).

O processo de auditoria do consumo de água em edificações subdivide-se em três fases. Inicialmente, é realizada uma análise e diagnóstico preliminar do consumo de água. Em seguida, um levantamento do edifício e diagnóstico do consumo. Por fim, as informações conduzem à elaboração de um plano de intervenção voltada à conservação de água na edificação analisada (SAUTCHUK et al., 2005). O processo de auditoria do consumo de água pode fazer uso de diferentes técnicas para obter informações sobre o uso da água na edificação analisada. Essas técnicas vêm sendo aprimoradas em função da necessidade do conhecimento detalhado do perfil do consumo de água, tanto na coleta quanto no tratamento dos dados (ROCHA; BARRETO e IOSHIMOTO, 1998).

Nesse sentido, este estudo teve como objetivo classificar e analisar as diferentes técnicas de auditoria do consumo de água em edificações. Para tanto, foi realizada uma revisão dos métodos delineados em estudos prévios que realizaram uma auditoria do consumo de água para caracterizar os usos finais de água, através de uma busca nos bancos de dados Scopus, SciELO, Web of Science e Google Scholar, utilizando as palavras-chave em Inglês foram "water audit", "water smart meter", "water end-use" e "water use". Os mesmos termos foram utilizados em português: "auditoria de água", "medidor de fluxo de água", "uso final de água" e "uso da água". A seleção dos artigos foi iniciada pelos títulos, em seguida pelos resumos e por fim, pela leitura do texto completo, para análise e exclusão daqueles não relacionados ao objetivo da pesquisa.

\section{TÉCNICAS DE AUDITORIA DO CONSUMO DE ÁGUA}

Foi possível observar o uso de diferentes técnicas de auditoria, desde a simples aplicação de questionários e registro de informações em diários (THACKRAY et al., 1978) ao monitoramento e aquisição de dados pela instalação de equipamentos de medição e registro de eventos de consumo em tempo real (DEOREO et al., 1996). As técnicas de auditoria do consumo de água em edificações podem ser classificadas em: 
a. Análise documental. Técnica que faz uso de contas de água, projetos hidráulicos e de arquitetura, ficha cadastral de moradores, funcionários, estudantes ou demais ocupantes, para coletar dados relativos ao consumo predial mensal e anual, composição das redes hidráulicas, área construída, área verde e população fixa e flutuante (OLIVEIRA, 1999). É uma técnica simples capaz de gerar indicadores de consumo de água per capita (litro/pessoa/dia) ou por área (litro $/ \mathrm{m}^{2} / \mathrm{dia}$ ).

b. Medições gerais. Técnica capaz de realizar o monitoramento do consumo de água a partir de medições pelo alimentador predial, podendo obter informações sobre o volume de água utilizado pela edificação dentro de um intervalo de tempo, pressão e detecção da existência de possíveis vazamentos. As leituras podem ser registradas à mão em um diário de registro (THACKRAY et al., 1978), remotamente por telemetria (TAMAKI et al., 2006) ou armazenadas em um dispositivo data logger (BARRETO, 2008).

c. Medições setorizadas. Consiste na instalação de medidores em determinados ambientes, unidades ou trechos hidráulicos para obtenção de informações relativas ao consumo de água para determinadas atividades (TAMAKI et al., 2006). Esta técnica é comumente associada ao uso de telemetria ou dispositivos data loggers para registro de dados.

d. Medições específicas. Utilizadas para quantificar os fluxos de água em pontos de consumo e podem empregar métodos variados. O mais simples, é pelo uso de cronômetros fixados ao lado de torneiras e chuveiros para a realização de registros do tempo de abertura de cada aparelho (SANT'ANA e MAZZEGA, 2017). Essa técnica é associada com diários para registrar, ao fim de cada dia, o tempo total de uso de água de cada ponto. $O$ volume de água utilizado pode ser estimado pelo produto do tempo registrado e vazão do aparelho. Sensores de fluxo instalados em pontos de uso de água realizam uma leitura instantânea do volume e do tempo de uso (ROCHA et al., 1998). Trata-se de um medidor de fluxo rotativo com sensor eletromagnético que emite um pulso a cada rotação da turbina e encaminha as informações para dispositivos data loggers para o armazenamento do registro de cada evento de uso de água. Um único medidor de fluxo pode ser instado no hidrômetro de entrada de uma rede direta de abastecimento de água para uma análise de traço da vazão - do Inglês, flow rate trace (DEOREO et al., 1996). Esta técnica consiste no rastreamento da assinatura da vazão de cada aparelho hidrossanitário, associado a um data logger para registro de dados e software capaz de identificar o perfil de consumo por uso final (GOMEZ; ALVES, 2000).

e. Diários de registro. Esta técnica envolve o registro de informações relativas ao uso ou consumo de água realizada por usuários ou gestores prediais, à mão ou por meios eletrônicos. O registro pode ser realizado em folhas fixadas ao lado de pontos de consumo (SANT'ANA, 2012), livro de registro de leituras de hidrômetro ou em diários individuais, um para cada usuário (GHISI e OLIVEIRA, 2006).

f. Observações. Técnica que possibilita examinar o comportamento de usuários e obter informações sobre seus hábitos de consumo de água e dos equipamentos utilizados. Por meio de observações diretas (in loco) dados de consumo podem ser extraídos no local onde os eventos de uso de água ocorrem naturalmente (NASCIMENTO e SANT'ANA, 2014), e devem ser realizadas com discrição de maneira a não interferir no 
comportamento do usuário para não resultar em informações incorretas (OLIVEIRA, 1999). Observações indiretas, faz uso de recurso audiovisual para registro de eventos de uso de água em ambientes em que a observação in loco não é possível, dada limitações espaciais (interferência do pesquisador nas atividades de usuários) e temporais (longos períodos de tempo para permanência de pesquisados) ou cujas ações de uso de água ocorrem simultaneamente (dificultando o registro por um único observador) (Sant'Anna et al., 2013).

g. Entrevistas. Técnica qualitativa capaz de extrair informações baseadas na percepção e vivência pessoal de usuários no levantamento das diferentes atividades relacionadas ao uso da água (SAUTCHUK et al., 2005). As entrevistas realizadas pelo pesquisador podem ser estruturadas (perguntas fechadas), semiestruturadas ou não-estruturadas (perguntas abertas).

h. Questionários. Técnica que consiste na elaboração de uma série de questões para coletar dados relativos ao uso de água de usuários com ou sem a presença do entrevistador, à mão ou por meios eletrônicos (KAMMERS e GHISI, 2005; CAMPUS et al., 2021, No prelo).

As técnicas de auditoria do consumo de água foram identificadas a partir da literatura revisada com uma amostragem de 37 artigos que abordaram 8 tipos de técnicas específicas, conforme apresentado na Tabela 1 e suas respectivas vantagens e desvantagens (Tabela 2). A Figura 1 apresenta a relação das técnicas de auditoria do consumo de água em edificações utilizadas pelos estudos analisados. Observa-se que mais de uma técnica pode ser utilizada por auditoria.

Figura 1 - Técnicas de auditoria utilizadas pelos estudos analisados

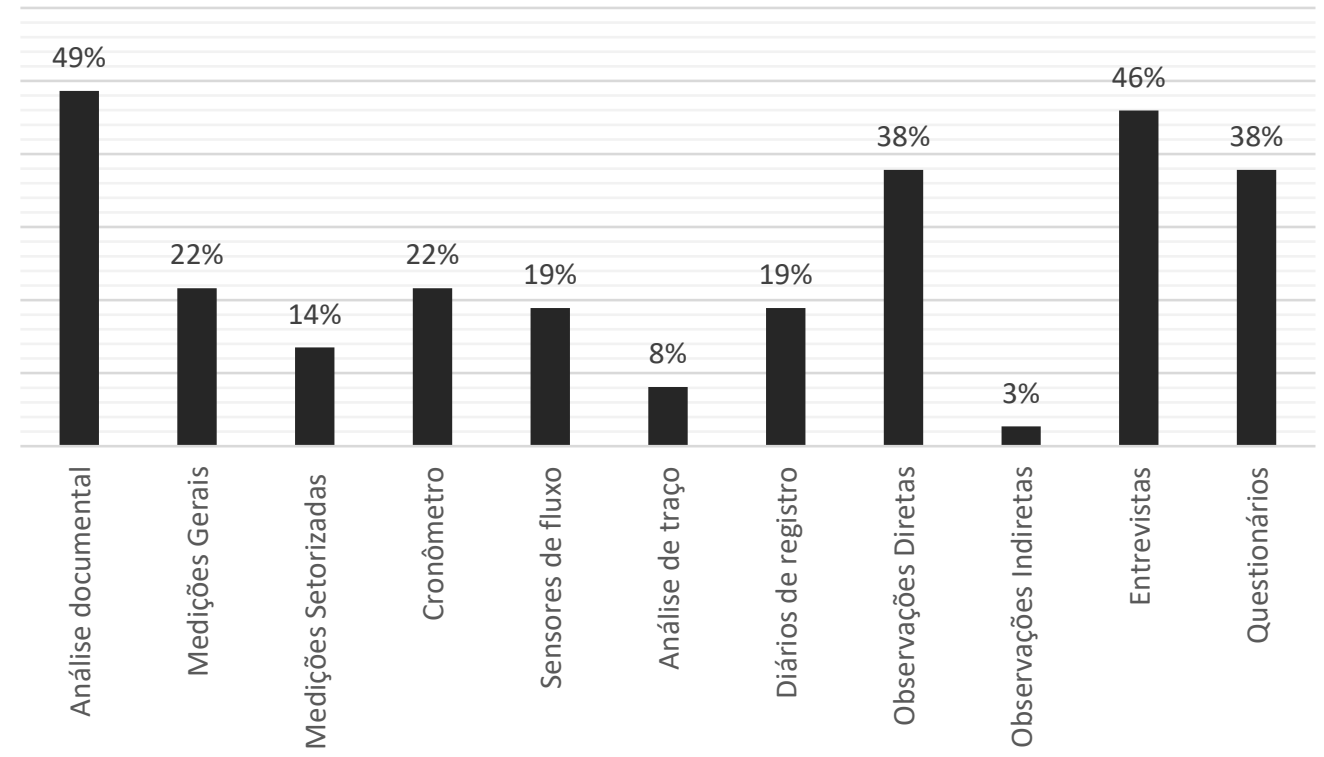

Fonte: Autoria própria. 
Tabela 1 - Técnicas de auditoria do consumo de água

\begin{tabular}{|c|c|c|c|c|c|c|c|c|c|c|}
\hline & \multirow{2}{*}{ 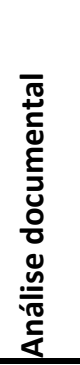 } & \multirow{2}{*}{ 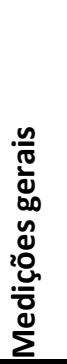 } & \multirow{2}{*}{ 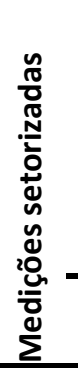 } & \multicolumn{2}{|c|}{ 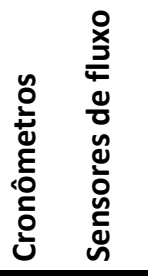 } & \multirow{2}{*}{ 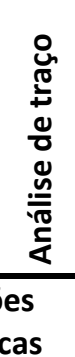 } & \multirow{2}{*}{ 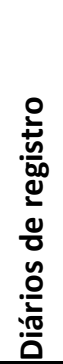 } & \multirow{2}{*}{ 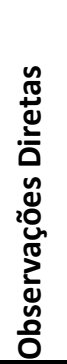 } & \multirow{2}{*}{ 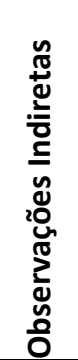 } & \multirow{2}{*}{ 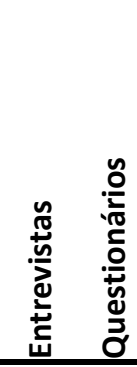 } \\
\hline & & & & & $\begin{array}{l}\text { lediçõ } \\
\text { pecífi }\end{array}$ & & & & & \\
\hline Thackray et al. (1978) & & $\theta$ & & & & & 8 & & & $\theta$ \\
\hline DeOreo et al. (1996) & & & & & & $\theta$ & $\theta$ & & & $\theta$ \\
\hline Rocha et al. (1998) & & $\theta$ & & & $\theta$ & & & & & \\
\hline Oliveira (1999) & 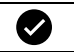 & $\theta$ & 8 & & & & & 8 & & \\
\hline Gomez e Alves (2000) & & & & & & $\theta$ & & $\theta$ & & 00 \\
\hline Cobacho et al. (2005) & & & 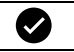 & & $\theta$ & & & & & \\
\hline Kammers e Ghisi (2005) & & & & & & & & & & 00 \\
\hline Ghisi e Oliveira (2006) & & & & & & & $\theta$ & & & \\
\hline Ilha et al. (2006) & & & & & & & & & & $\theta$ \\
\hline Barreto (2008) & & $\theta$ & & & $\theta$ & & & & & \\
\hline Marinoski e Ghisi (2008) & & & & & & & & & & 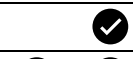 \\
\hline Proença e Ghisi (2009) & & & & & & & & & & 00 \\
\hline Fasola et al. (2011) & & & & & & & & & & 00 \\
\hline Sant'Ana (2012) & 8 & & & $\theta$ & & & $\theta$ & $\theta$ & & $\theta$ \\
\hline Calza et al. (2012) & 8 & & & & & & & & & \\
\hline Agana et al. (2013) & & & $\theta$ & & & & & & & \\
\hline Barrington et al. (2013) & & & & & & & & & $\theta$ & 0 \\
\hline Bint et al. (2013) & 8 & & & & $\theta$ & & & & & \\
\hline Sant'Ana et al. (2013) & 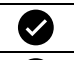 & & $\theta$ & 2 & $\theta$ & & $\theta$ & $\theta$ & & $\theta$ \\
\hline Sant'Anna et al. (2013) & 8 & $\theta$ & & $\theta$ & & & 8 & 8 & & $\theta$ \\
\hline Nascimento e Sant'Ana (2014) & & & & $\theta$ & & & & 8 & & 00 \\
\hline Collett et al. (2016) & & & & & & & & 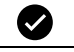 & & \\
\hline Jenicek et al. (2016) & & & & & & $\theta$ & & & & $\theta$ \\
\hline D'Alessandro et al. (2016) & & & & & & & & & & 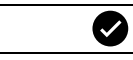 \\
\hline Khan (2016) & & & & $\theta$ & & & & $\theta$ & & \\
\hline García-Sanz-Calcedo et al. (2017) & & $\theta$ & & & & & & & & \\
\hline Santana e Sant'Ana (2017) & & & & 8 & & & & $\theta$ & & 2 \\
\hline Sant'Ana e Mazzega (2017) & $\theta$ & $\theta$ & & $\theta$ & & & $\theta$ & & & 00 \\
\hline Soares et al. (2017) & & & 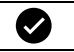 & & & & & $\theta$ & & $\theta$ \\
\hline Barbosa et al. (2018) & 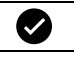 & & & & & & & $\varnothing$ & & 00 \\
\hline Buono (2018) & & & & & & & & & & $\theta$ \\
\hline Gómez-Chaparro et al. (2018) & & $\varnothing$ & & & & & & & & $\theta$ \\
\hline Santos e Sant'Ana (2019) & & & & 2 & & & & 8 & & \\
\hline Miranda et al. (2019) & & & & & $\theta$ & & & & & 00 \\
\hline Potgieter et al. (2020) & & & & & & & & 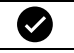 & & \\
\hline Trindade et al. (2020) & & & & & & & & 8 & & $\theta$ \\
\hline Bomfim e Sant'Ana (2021) & & & & & & & & & & \\
\hline
\end{tabular}

Fonte: Autoria própria. 
Tabela 2 - Vantagens e desvantagens

\begin{tabular}{|c|c|c|}
\hline TÉCNICA & VANTAGENS & DESVANTAGENS \\
\hline Análise documental & $\begin{array}{l}\text { - Informações gerais do consumo de } \\
\text { água predial }\end{array}$ & $\begin{array}{l}\text { - Informações sobre consumo não } \\
\text { correspondem ao período de auditoria } \\
\text { - Não fornece informações sobre uso final } \\
\text { de água }\end{array}$ \\
\hline Medições Gerais & $\begin{array}{l}\text { - Medições gerais do consumo de água } \\
\text { predial }\end{array}$ & $\begin{array}{l}\text { - Não fornece informações sobre uso final } \\
\text { de água } \\
\text { - Acompanha as alterações do consumo de } \\
\text { água na edificação }\end{array}$ \\
\hline Medições Setorizadas & $\begin{array}{l}\text { - Medições precisas do consumo de } \\
\text { água em setores específicos } \\
\text { - Identificação de possíveis vazamentos }\end{array}$ & $\begin{array}{l}\text { - Técnica mais complexa e demorada } \\
\text { - Necessária a instalação de vários } \\
\text { hidrômetros } \\
\text { - Custo elevado quando comparado a } \\
\text { outras técnicas } \\
\text { - Identificação dos usos da água por setor }\end{array}$ \\
\hline \multirow[t]{6}{*}{ Medições Específicas } & Cronômetros & \\
\hline & $\begin{array}{l}\text { - Informações do tempo de uso dos } \\
\text { aparelhos sanitários } \\
\text { - Medição do volume de água } \\
\text { - Técnica versátil }\end{array}$ & $\begin{array}{l}\text { - Necessária a realização de levantamento } \\
\text { dos equipamentos consumidores de água } \\
\text { para cálculo da vazão }\end{array}$ \\
\hline & Sensores de fluxo & \\
\hline & $\begin{array}{l}\text { - Técnica precisa e confiável } \\
\text { - Detalhamento do consumo de água } \\
\text { por aparelho sanitário } \\
\text { - Informações sobre o uso final de água } \\
\text { - Capacidade de medição de vazão com } \\
\text { precisão } \\
\text { - Técnica indicada para qualquer } \\
\text { tipologia }\end{array}$ & $\begin{array}{l}\text { - Técnica mais complexa e demorada } \\
\text { - Custo elevado quando comparado a } \\
\text { outras técnicas }\end{array}$ \\
\hline & Análise de traço & \\
\hline & $\begin{array}{l}\text { - Técnica não invasiva } \\
\text { - Nível de precisão satisfatório para } \\
\text { determinação do perfil do uso de água } \\
\text { - Informações sobre uso final de água }\end{array}$ & $\begin{array}{l}\text { - Restrição quanto ao rastreio do consumo } \\
\text { de água quando há utilização simultânea } \\
\text { de mais de um equipamento } \\
\text { - Limitação técnica para edifícios com } \\
\text { alimentação indireta, com reservatório } \\
\text { elevado } \\
\text { - Técnica mais adequada para edificações } \\
\text { residenciais }\end{array}$ \\
\hline Diários de Registro & $\begin{array}{l}\text { - Economia de custo para realização da } \\
\text { auditoria } \\
\text { - Informações sobre o uso final de água } \\
\text { - Técnica simples }\end{array}$ & $\begin{array}{l}\text { - Necessário o registro o do tempo e } \\
\text { frequência de uso pelo usuário } \\
\text { - Técnica passível de erro } \\
\text { - Precisam ser bem elaborados para } \\
\text { obtenção dos dados }\end{array}$ \\
\hline
\end{tabular}


Tabela 2 - Vantagens e desvantagens (Continuação)

\begin{tabular}{|c|c|c|}
\hline TÉCNICA & VANTAGENS & DESVANTAGENS \\
\hline \multirow[t]{4}{*}{ Observações } & Diretas (in loco) & \\
\hline & $\begin{array}{l}\text { - Possibilita avaliar o comportamento } \\
\text { dos usuários quanto ao uso da água } \\
\text { - Permite a obtenção de dados não } \\
\text { possíveis com uso do questionário e da } \\
\text { entrevista } \\
\text { - Não há um roteiro para a coleta de } \\
\text { dados } \\
\text { - Permite registrar o tempo e } \\
\text { frequência de uso de cada aparelho } \\
\text { sanitário } \\
\text { - Identificação do perfil do consumo de } \\
\text { água }\end{array}$ & $\begin{array}{l}\text { - Técnica mais demorada } \\
\text { - O usuário tende a criar impressões } \\
\text { desfavoráveis pela presença do observador } \\
\text { - Os usos dos aparelhos sanitários podem } \\
\text { ocorrer simultaneamente, dificultando a } \\
\text { coleta de dados }\end{array}$ \\
\hline & Indiretas (vídeo) & \\
\hline & $\begin{array}{l}\text { - Possibilita avaliar o comportamento } \\
\text { dos usuários quanto ao uso da água } \\
\text { - Permite a obtenção de dados não } \\
\text { possíveis com uso do questionário e da } \\
\text { entrevista } \\
\text { - Não há um roteiro para a coleta de } \\
\text { dados } \\
\text { - Permite registrar o tempo e } \\
\text { frequência de uso de cada aparelho } \\
\text { sanitário } \\
\text { - Identificação do perfil do consumo de } \\
\text { água }\end{array}$ & $\begin{array}{l}\text { - Técnica mais rápida e eficiente } \\
\text { - O usuário não cria impressões } \\
\text { desfavoráveis porque não há a presença do } \\
\text { observador no local } \\
\text { - Permite a coleta de dados quando há uso } \\
\text { simultâneo dos aparelhos sanitários }\end{array}$ \\
\hline Entrevistas & $\begin{array}{l}\text { - Informações sobre hábitos do } \\
\text { consumo de água } \\
\text { - Levantamento da frequência e tempo } \\
\text { de uso dos aparelhos sanitários } \\
\text { - Quantificação do número de } \\
\text { aparelhos sanitários e características } \\
\text { tipológicas }\end{array}$ & $\begin{array}{l}\text { - Disposição do entrevistado em fornecer } \\
\text { informações necessárias } \\
\text { - Necessidade de tempo para realização }\end{array}$ \\
\hline Questionários & $\begin{array}{l}\text { - Informações sobre hábitos do } \\
\text { consumo de água } \\
\text { - Levantamento da frequência e tempo } \\
\text { de uso dos aparelhos sanitários } \\
\text { - Identificação do número de aparelhos } \\
\text { sanitários e características tipológicas } \\
\text { das edificações } \\
\text { - Economiza tempo e obtém grande } \\
\text { número de dados } \\
\text { - Atinge um maior número de pessoas } \\
\text { simultaneamente } \\
\text { - Economia de pessoal no trabalho de } \\
\text { campo }\end{array}$ & $\begin{array}{l}\text { - Inconveniente para usuários dependendo } \\
\text { da tipologia } \\
\text { - Porcentagem pequena dos questionários } \\
\text { que voltam } \\
\text { - Grande número de perguntas sem } \\
\text { resposta } \\
\text { - Depende da assiduidade das anotações } \\
\text { pelo usuário } \\
\text { - Depende do entendimento do usuário, } \\
\text { da sistemática das anotações relacionadas } \\
\text { aos usos da água, horários, frequências e } \\
\text { dos dados provenientes da caracterização } \\
\text { dos aparelhos sanitários } \\
\text { - Impossibilidade de ajudar o entrevistado } \\
\text { em questões mal compreendidas }\end{array}$ \\
\hline
\end{tabular}




\section{CONSIDERAÇÕES FINAIS}

A auditoria do consumo de água é considerada uma prática desejável para propor estratégias conservadoras e evitar problemas futuros com a falta de água nas edificações. Considerando a necessidade de estimar onde e como a água é usada no edifício, o uso de diferentes técnicas auxilia na busca dessas informações e as vantagens e desvantagens identificadas nesse processo de revisão de literatura podem ser consideradas como fatores para a escolha da técnica a ser empregada.

Verificou-se que a análise documental pode não corresponder ao período da realização da auditoria do consumo de água, que as medições no hidrômetro fornecem apenas o consumo predial total e as medições setorizadas, o consumo de água por setores em que os hidrômetros foram instalados.

Técnicas como entrevistas, questionários, observações e diários de registro auxiliam nas estimativas dos usos finais, mas podem gerar informações imprecisas. Por sua vez, as medições específicas fornecem frequência e tempo de uso de cada aparelho sanitário, bem como os usos finais de água. É uma técnica mais precisa, porém mais complexa e demorada, além da necessidade da instalação de equipamentos específicos para medição do consumo de água. A maioria dos estudos na literatura revisada utilizou análise documental (49\%), entrevistas (46\%), questionários (38\%) e observações diretas (38\%). Poucos fizeram uso de técnicas mais precisas para estimar os usos finais de água utilizando sensores de fluxo (19\%) ou análise de traço (8\%).

\section{AGRADECIMENTOS}

Ao CNPq e à CAPES, pelo apoio recebido e à Universidade de Brasília, pelo financiamento proveniente da FUB/UnB/DPG para apoio à execução de projetos de pesquisas científicas, tecnológicas e de inovação.

\section{REFERÊNCIAS}

AGANA, B. A.; REEVE, D.; ORBELL, J. D. An approach to industrial water conservation - A case study involving two large manufacturing companies based in Australia. Journal of Environmental Management, v. 114, p. 445-460, 2013.

ALCANTARA, I.; Técnicas de auditoria do consumo de água: relatos de experiência em campo. In: PANTOJA, João da Costa; BUZAR, Márcio Augusto Roma; PORTO, Naiara Guimarães de Oliveira (org.). Tecnologia, ambiente e sustentabilidade: coletânea de artigos. p. 142-156. Brasília: LaSUS FAU, 2021.

BARBOSA, G. G.; BEZERRA, S. P.; SANT'ANA, D. Indicadores de consumo de água e análise comparativa entre o aproveitamento de águas pluviais e o reúso de águas cinzas em edificações de ensino do Campus Darcy Ribeiro - UnB. Paranoá, Brasília, n. 22, p. 1-15, 2018.

BARRETO D. Perfil do consumo residencial e usos finais da água. Ambiente Construído, Porto Alegre, v. 8, n. 2, p. 23-40, abr./jun. 2008.

BARRINGTON, D. J.; PRIOR, A.; HO, G. The role of water auditing in achieving water conservation in the process industry. Journal of Cleaner Production, v. 52, p. 356-361, 2013.

BINT, L.; VALE, R.; ISAACS, N. Water Efficiency in Office Buildings. Water Efficiency in Buildings: Theory 
and Practice, p. 241-251, 2013.

BOMFIM, B. C.dos S.; SANT'ANA, D. Análise dos usos-finais de água de uma quitinete em Brasília. In: SANT'ANA, Daniel (org.). Água e o ambiente construído. Ponta Grossa - PR: Atena, 2021. p. 25-36.

BUONO, L. N. Diagnóstico de uso de água no hospital de Londrina : estudo de caso. Londrina: Dissertação (Mestrado em Engenharia Ambiental) - Universidade Tecnológica Federal do Paraná, 2018.

CALZA, L. F.; NOGUEIRA, C. E. C.; SIQUEIRA, J. A. C. Diagnóstico preliminar e proposta de adequação para o uso eficiente da água no hospital universitário do oeste do Paraná. Acta Iguazu, v. 1, n. 4, p. $27-$ 35, 2012.

CAMPUS, M. A. S. et al. Impact of the COVID-19 pandemic on water consumption behaviour. Water Supply, v?, n?, p. 1-10, 2021. No prelo.

COBACHO, R. et al. Improving efficiency in water use and conservation in Spanish hotels. Water Science and Technology: Water Supply, v. 5, n. 3-4, p. 273-279, 2005.

COLLETT, S. et al. Water usage in a multi-speciality hospital and its effective management. Journal of The Academy of Clinical Microbiologists, v. 18, n. 2, p. 135, 2016.

D'ALESSANDRO, D. et al. Water use and water saving in Italian hospitals: A preliminary investigation. Annali dell'Istituto Superiore di Sanità, v. 52, p. 56-62, 2016.

DEOREO, W. B.; HEANEY, J. P.; MAYER, P. W. Flow trace analysis to assess water use. American Water Works Association, v. 88, p. 79-90, 1996.

FASOLA, G. B. et al. Potencial de economia de água em duas escolas em Florianópolis, SC. Ambiente Construído, v. 11, n. 4, p. 65-78, 2011.

GARCÍA SANZ-CALCEDO, J. et al. Analysis of the average annual consumption of water in the hospitals of extremadura (Spain). Energies, v. 10, n. 4, 2017.

GHISI, E.; OLIVEIRA, S. M. DE. Potential for potable water savings by combining the use of rainwater and greywater in houses in southern Brazil. Building and Environment, v. 42, n. 4, p. 1731-1742, 2006.

GÓMEZ-CHAPARRO, M.; GARCÍA SANZ-CALCEDO, J.; ARMENTA-MÁRQUEZ, L. Study on the use and consumption of water in Spanish private hospitals as related to healthcare activity. Urban Water Journal, v. 15, n. 6, p. 601-608, 2018.

GOMEZ, J.; ALVES, W. C. Final Water Consumption in Building Installations Using the Flow Rate Trace. In: Proceedings 26th International Symposium on Water Supply and Drainage for Buildings. n. 1, p. 19, 2000.

HONORATO, K. M.; CAMPOS, M. A. S. Análise das vazões de projeto de água fria em uma residência unifamiliar: Comparação entre métodos empíricos e probabilísticos. Paranoá n. 27, p. 59-78, 2019.

ILHA, M. S. D. O.; NUNES, S. DA S.; SALERMO, L. S. Programa de conservação de água em hospitais: estudo de caso do Hospital das Clínicas da Universidade Estadual de Campinas. Ambiente Construído, v. 6, n. 1, p. 91-97, 2006.

JENICEK, E. M. et al. Methods of Estimating Water End Use at US Army Installations. Journal - American Water Works Association, v. 108, n. 2, p. E67-E76, 2016. 
KAMMERS, P. C.; GHISI, E. Usos finais de água em edifícios públicos localizados em Florianópolis, SC. Ambiente Construído, v. 6, n. 1, p. 75-90, 2005.

KHAN, S. Restroom water reduction potential at an Urban University. Sustainability (United States), v. 9, n. 6, p. 295-304, 2016.

MARINOSKI, A. K.; GHISI, E. Aproveitamento de água pluvial para usos não potáveis em instituição de ensino: estudo de caso em Florianópolis - SC. Ambiente Construído, v. 8, n. 2, p. 67-84, 2008.

MAZZEGA, P. ; LE QUEAU, D. ; SIBERTIN-BLANC, C. ; SANT'ANA, D. Water Management and Development: The Limits of Coordination.. In: Boulet R.; Lajaunie C.; Mazzega P. (Org.). Law, Public Policies and Complex Systems: Networks in Action. 1ed.: Springer, 2019, v. 42, p. 153-175.

NASCIMENTO, E. A. A.; SANT'ANA, D. Caracterização dos Usos-Finais do Consumo de Água em Edificações do Setor Hoteleiro de Brasília. Revista de Arquitetura IMED, v. 3, n. 2, p. 156-167, 2014.

OLIVEIRA, L. H. Metodologia para a implantação de programa de uso racional da água em edifícios. São Paulo: Tese (Doutorado em Engenharia) - Universidade de São Paulo, 1999.

POTGIETER, M. S. W. et al. Water-wise hand preparation-the true impact of our practice: A controlled before-and-after study. South African Medical Journal, v. 110, n. 4, p. 291-295, 2020.

PROENÇA, L. C.; GHISI, E. Estimativa de usos finais de água em quatro edifícios de escritórios localizados em Florianópolis. Ambiente Construído, v. 9, n. 3, p. 95-108, 2009.

ROCHA, A. L.; BARRETO, D.; IOSHIMOTO, E. Caracterização e monitoramento do consumo predial de água. p. 38, 1998.

SANT'ANA, D. Domestic Water end-uses and water conservation in multistorey buildings in the Federal District, Brazil. In: PLEA 2012 - 28th Conference, Opportunities, Limits \& Needs Towards an environmentally responsible architecture Lima, Perú, 2012.

SANT'ANA, D.; BOEGER, L.; MONTEIRO, L. Aproveitamento de águas pluviais e o reúso de águas cinzas em edifícios residenciais de Brasília - parte 1: reduções no consumo de água. Paranoá, Brasília, v. 10, n. 10, p. 77-84, 2013.

SANT'ANA, D.; MAZZEGA, P. Socioeconomic analysis of domestic water end-use consumption in the Federal District, Brazil. Sustainable Water Resources Management, v. 4, n. 4, p. 921-936, 2018.

SANTANA, P. M.; SANT'ANA, D. Water use and conservation in educational centres of the Federal District, Brazil. Proceedings of 33rd PLEA International Conference: Design to Thrive, PLEA 2017, v. 3, n. July, p. 5173-5180, 2017.

SANT'ANNA, R.; MIRANDA, R.; CÉSAR, L.; SANT'ANA, D. Análise do consumo de água em escola pública do Distrito Federal. In: SEABRA, Giovanni (org.). Terra: qualidade de vida, mobilidade e segurança nas cidades. João Pessoa: Editora Universitária UFPB, 2013. v. 3, p. 1231-1243.

SANTOS, P. A. S.; RAMOS, S. R.; SANT'ANA, D. Uso racional de água: análise do potencial de redução do consumo em escolas públicas. In: III Encuentro Latinoamericano y Europeo sobre Edificaciones y Comunidades Sostenibles, 2019.

SAUTCHUK, C. et al. Conservação e Reúso da água em Edificações. São Paulo: Prol Editora Gráfica, 2005. 
SOARES, A. E. P. S.; NUNES, L. G. C. F.; SILVA, S. R. DA. Diagnóstico dos Indicadores de Consumo de Água em Escolas Públicas de Recife - PE. Fórum Ambiental da Alta Paulista, v. 13, n. 1, p. 107-118, 2017.

TAMAKI, H.O.; Silva, G. S.; Gonçalves, O. M. A medição setorizada como instrumento de gestão da demanda de água em sistemas prediais. Ambiente Construído, v. 6, n.1, p. 63-74, 2006.

THACKRAY, J. E.; COCKER, V.; ARCHIBALD, G. The Malvern and Mansfield studies of domestic water usage. Proceedings of the institution of civil engineers: part 1 - design and construction, v. 64, p. 3761, 1978.

TRINDADE, M.; BUONO, L. N.; COSTANZI, R. N. Uso de água em lavanderia de Hospital Universitário de Londrina: estudo de caso. Revista Gestão \& Sustentabilidade Ambiental, v. 9, p. 921, 2020. 